\title{
Distributed Stream Consistency Checking
}

Gao, Shen ; Dell'Aglio, Daniele ; Pan, Jeff Z ; Bernstein, Abraham

DOI: https://doi.org/10.1007/978-3-319-91662-0_32

Posted at the Zurich Open Repository and Archive, University of Zurich

ZORA URL: https://doi.org/10.5167/uzh-152208

Conference or Workshop Item

Accepted Version

Originally published at:

Gao, Shen; Dell'Aglio, Daniele; Pan, Jeff Z; Bernstein, Abraham (2018). Distributed Stream Consistency Checking. In: Web Engineering - 18th International Conference, ICWE 2018, Cáceres, Spain, June 5-8, 2018, Proceedings, Cáceres, Spain, 5 June 2018 - 8 June 2018. Springer, 387-403.

DOI: https://doi.org/10.1007/978-3-319-91662-0_32 


\title{
Distributed Stream Consistency Checking
}

\author{
Shen Gao ${ }^{1}$, Daniele Dell'Aglio ${ }^{1}$, Jeff Z. Pan ${ }^{2}$, and Abraham Bernstein ${ }^{1}$ \\ 1 DDIS, Department of Informatics, University of Zurich, Switzerland \\ [shengao, dellaglio, bernstein] @ifi.uzh.ch \\ 2 The University of Aberdeen, United Kingdom \\ jeff.z.pan@abdn.ac.uk
}

\begin{abstract}
Dealing with noisy data is one of the big issues in stream processing. While noise has been widely studied in settings where streams have simple schemas, e.g. time series, few solutions focused on streams characterized by complex data structures. This paper studies how to check consistency over large amounts of complex streams. Our proposed methods exploit reasoning to assess if portions of the streams are compliant to a reference conceptual model. To achieve scalability, our methods runs on state-of-the-art distributed stream processing platforms, e.g. Apache Storm or Twitter Heron. Our first method computes the closure of Negative Inclusions (NIs) for DL-Lite ontologies and registers the NIs as queries. The second method compiles the ontology into a processing pipeline to evenly distribute the workload. Experiments compares the two methods and show that the second one improves the throughput up to $139 \%$ with the LUBM ontology and $330 \%$ with the NPD ontology.
\end{abstract}

\section{Introduction}

Web data streams are changing our lives. Continuously generated news streams transform search engines in up-to-date newspapers, where breaking news is listed at the top of query results. City-related sensor streams improve our driving experience, with real-time information about navigation, traffic, and events. Recently, stream reasoning [22,23] is also considered in Knowledge Graph [18, 17], where frequent (or even constant) updates of knowledge graphs become a norm.

However, noise can lead to wrong and unexpected results, e.g. errors in traffic sensors lead to wrong representations of the current status of a city, causing - in the worst case - new jams. It is worth noting that noise is inevitable in streaming settings, so technological solutions to cope with it are necessary. A possible way to handle noise is through data integrity constraints, such as data range filters that exclude outliers generated by sensors with hardware failures. However, those methods may fail in detecting noise when data is described according to complex conceptual models. The problem we study in this article is how to assess the consistency of streams w.r.t. a fixed and known a-priori conceptual model.

In stream processing scenarios, an important requirement is responsiveness: the consistency assessment process must be responsive and quick when analyzing newly arrived data. Batch-based solutions are generally not efficient due to 
the high latency involved. Furthermore, they have the overhead of processing overlapped data between batches multiple times. Therefore, the stream scenario calls for an incremental solution to cope with the velocity dimension.

The first challenge we cope with is: how to model the problem of stream consistency checking? Checking if a (static) dataset is consistent w.r.t. a schema is a well-known problem in data and knowledge management $[2,9,16]$. Moving to the streaming setting, consistency checks in presence of data streams has only been partially considered so far $[22,28]$. Such studies assume that the input stream can be managed as a whole. This creates a problem w.r.t. responsiveness, since the whole input stream is needed to produce a result. It follows that methods that compute the output incrementally are desirable in this setting.

In this paper, we consider the DL-lite ${ }_{\text {core }}$ description logic to express the

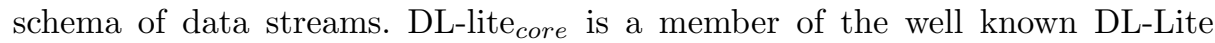
family, which provides the underpinnings for the standard ontology language OWL2 QL.These logics are expressive enough to cover most features in UML diagrams, and their data complexity for the conjunctive query answering task is $A C_{0}$, which is the same as conjunctive querying over relational databases.

The second problem arises from the volume of the data: if we consider the set of streams as a whole, the size of the data may be too large to fit in one computing node. We adopt a usual assumption in stream processing settings: the more recent the data, the more relevant it is. This translates in the introduction of windows to capture recent portions of the stream. However, even in this way, the volume of the data may still be too big to be treated in a single machine. A way to overcome this limitation is to perform the consistency check in a distributed fashion. Therefore, a key question is how to distribute the stream consistency reasoning task while guaranteeing correctness? This allows exploiting a Distributed Stream Processing Engines (DSPEs), e.g. Apache Flink and Twitter Heron, which require users to compile the business logics into processing workflows. We focus on how to assess stream consistency on top of DSPEs.

Summarising, the main contributions of this paper include:

1. a formalisation of the stream consistency checking problem w.r.t. a static DL-lite core $_{\text {conceptual schema; }}$

2. two scalable and incremental methods for stream consistency checking. They are designed for a distributed environment. Intermediate reasoning results are cached in the system to check the consistency for newly arrived streams;

3. an analysis of these methods through comparative studies based on the LUBM benchmark [11] and the NPD [26] ontology using Twitter Heron.

\section{Preliminaries}

In this section we introduce DL-lite core $_{\text {as }}$ the Description Logic (DL) we consider in this study. We then describe evolving ontologies, and finally we discuss the distributed stream processing concepts we used to build our solutions. 
Static DL-lite core $_{\text {Ontology. DL-lite }}$ core is a description logic of the DL-Lite family [3]. Its building blocks are named concepts (denoted with $A$ ) and named roles (denoted with $P$ ). They can be used to build basic concepts $C$ as $C$ := $\perp|A| \exists R$; and basic roles $R$ as $R:=P \mid P^{-}$. A DL-lite core $_{\text {contology is }}$ composed of a TBox $\mathcal{T}$ and an ABox $\mathcal{A}$. The TBox $\mathcal{T}$ contains axioms in the forms of $C_{1} \sqsubseteq C_{2}$ or $C_{1} \sqsubseteq \neg C_{2}$. The former axiom, also known as Positive Inclusion (PI), indicates that $C_{1}$ is a subclass of $C_{2}$, e.g. Student is a subclass of Person (also denoted $S C$ (Student,Person)). The latter one represents a Negative Inclusion (NI), which expresses that two classes are disjoint, e.g. there cannot be an instance of Person and Organization (also denoted $D J\left(C_{1}, C_{2}\right)$ ).

The ABox $\mathcal{A}$ contains assertions about individuals, which can be either $C_{k}\left(x_{1}\right)$ or $R_{k}\left(x_{1}, x_{2}\right)$, where $x_{1}$ and $x_{2}$ are two individual instances. If an NI in $\mathcal{T}$ is violated by assertions of $\mathcal{A}$, (e.g. $D J\left(C_{1}, C_{2}\right)$ in $\mathcal{T}, C_{1}(a)$ and $C_{2}(a)$ in $\mathcal{A}$ ), then the knowledge base is inconsistent. [6] shows that only NI axioms

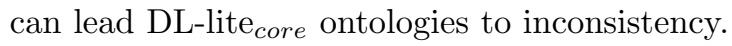

$R D F$ and DL ontology streams. An RDF stream $S=\left(\left(d_{1}, t_{1}\right), \ldots,\left(d_{n}, t_{n}\right), \ldots\right)$ is a potentially unbounded sequence of timestamped informative units $\left(d_{i}, t_{i}\right)$ ordered by the temporal dimension, where $t_{i}$ is the timestamp (we consider the time as discrete) and $d_{i}$ is an RDF statement. An RDF statement is a triple $(s, p, o) \in(I \cup B) \times I \times(I \cup B \cup L)$, where $I, B$, and $L$ identify the sets of IRIs, blank nodes and literals, respectively. An $R D F$ term is an element of the set $T=I \cup B \cup L$. RDF streams can be used to serialise DL ontology streams.

We define DL ontology streams starting from the notion of evolving ontology as defined in $[22,15]$, which captures the dynamics of a knowledge base. Given a discrete time interval $[m, n]$, a DL ontology stream $\mathcal{O}_{m}^{n}$ is a pair $\mathcal{O}_{m}^{n}=\left(\mathcal{T}, \mathcal{A}_{m}^{n}\right)$, where $\mathcal{T}$ is a TBox and $\mathcal{A}_{m}^{n}$ is a stream of ABoxes from time $m$ to $n$. We refer with $\mathcal{A}_{m}^{n}(i)$ to the ABox (snapshot) at time $i$ associated to $\mathcal{A}_{m}^{n}$. Similarly, we refer with $\mathcal{O}_{m}^{n}(i)$ to the pair $\left(\mathcal{T}, \mathcal{A}_{m}^{n}(i)\right)$, which is a static ontology.

When handling a stream, it may be important to analyze a portions of data items, e.g., count the occurrences of a fact over a time interval. Inspired by the research on stream and event processing [8], we employ the notion of window, which is an operation that selects a portion of items in the stream. Let $\mathcal{O}_{m}^{n}$ be a DL ontology stream. The application of a window $\mathrm{W}$ over $\mathcal{O}_{m}^{n}$ results in:

$\mathcal{O}_{o}^{c}=W\left(\mathcal{O}_{m}^{n}, \omega, c\right)=\left(\mathcal{O}_{m}^{n}(o), \ldots, \mathcal{O}_{m}^{n}(c)\right)=\left(\mathcal{T},\left\langle\mathcal{A}_{m}^{n}(o), \ldots, \mathcal{A}_{m}^{n}(c)\right\rangle\right)=\left(\mathcal{T}, \mathcal{A}_{o}^{c}\right)$,

where $\omega$ is a natural number representing the size of the window, and $c$ is the time on which the window is applied. The following constraints hold: $o=$ $\max \{m, c-\omega\}$ and $c \leq n$. Finally, we define the window content as the union of the axioms contained in the stream snapshots, i.e., $u\left(\mathcal{A}_{m}^{n}\right)=\bigcup_{i=m}^{n} \mathcal{A}_{m}^{n}(i)$. Given a DL ontology stream $\mathcal{O}_{m}^{n}$, it follows that $\left\langle\mathcal{T}, u\left(\mathcal{A}_{m}^{n}\right)\right\rangle$, is a knowledge base.

Distributed Stream Processing Engines. Processing big amounts of streams usually relies on Distributed Stream Processing Engines (DSPEs). DSPEs provide the advantages of automatic instantiating and distributing tasks onto computing 

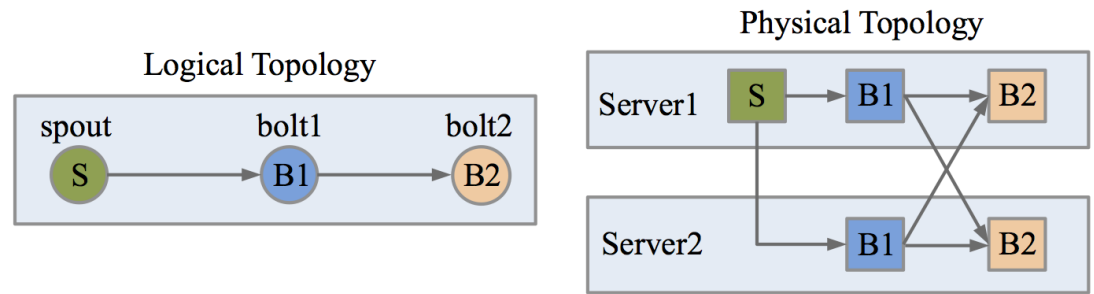

Fig. 1: Deploying a logical topology (left) into a physical (right) one (right). The spout $S$ and bolts $B 1, B 2$ are instantiated as tasks on two computing servers.

nodes. They also take care of the coordination of the tasks. Users just need to provide the processing logics. In the following, we introduce basic concepts of DSPE, adopting the nomenclature of Apache Storm and Heron.

Fig. 1 (left) shows the processing logics as a logical topology, which is a Directed Acyclic Graph (DAG) composed of spout and bolt nodes. Spouts $(S)$ provide input data to bolts; they emit data to downstream nodes and are typically used to connect a topology with external data sources such as Web services or data brokers. Bolts $\left(B_{i}\right)$ embed the processing logics: they manipulate data from upstream nodes and emit results to downstream nodes.

Each edge of the topology represents a data stream. Data streams flow through the topology as sequences of tuples, i.e. sets of property-value pairs. While defining the logical topology, the user should declare the tuple format (i.e., the set of properties) for every edge.

In addition to the structure of the logical topology, the user should provide configuration information to deploy the logical topology to a computing cluster. First, the user needs to define how many instances of each node should be deployed. These instances are named task instances or, shortly, tasks. Moreover, for each edge in the logical topology, the user should define a grouping strategy (e.g., a hashing function), which is used by the DSPE to partition the stream among the tasks. For this reason, given a stream, a subset of the tuple attributes acts as a key. The DSPE uses the logical topology and the configuration parameters to decide how to distribute the tasks among the available computing servers. This results in a physical topology, as depicted on the right of Fig. 1.

Problem definition. We study how to perform incremental stream consistency

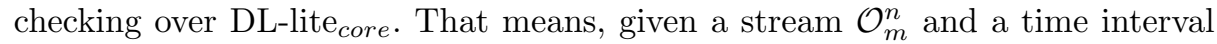
of $\omega$ time units, we aim to check if $\left\langle\mathcal{T}, u\left(W\left(\mathcal{A}_{m}^{n}, \omega, c\right)\right)\right\rangle$ is consistent for every $c \in[m, n]$ in an incremental way. It is worth noting that when $\omega$ is large and the window starts at the beginning of the input (i.e., $\omega \geq n-m$ and $m=0$ ), the problem becomes the incremental consistency assessment over the whole stream. 


\section{Related Work}

We first discuss related studies on consistency checking for knowledge bases. Then, we introduce distributed stream processing and RDF stream processing.

Consistency Checking of an Ontology. Although there have been many studies on consistency checking, e.g., Baclawski et al. [4] translate an ontology to the language that can be executed by a logic programming engine, stream consistency checking is only partially considered. [28] proposes an incremental method that efficiently checks the consistency of new changes against the whole ontology.

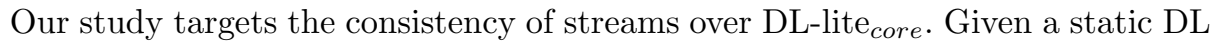
ontology, consistency checking is often reduced to query answering. There are various query rewriting techniques that have been proposed $[6,12,21]$. Specially, [6] shows that the computational complexity of a consistency checking task is exponential to the size of TBox at worst. Recently, [19,20] proposed the adoption of machine learning to achieve fact consistency checking. These methods apply standard reasoning techniques to label inconsistent data instances, and then learn a model to classify new instances. While these approaches are interesting, they cannot guarantee a $100 \%$ accuracy. When considering dynamic ontologies, there are some studies on incremental reasoning and evolving ontologies $[24,15]$. However, they do not consider distributed stream settings.

Distributed Stream Processing. Stream processing relies on the idea of managing data in motion, by performing tasks in a continuous fashion. This paradigm has recently gained popularity due to the rise of Distributed Stream Processing Engines (DSPEs), which process streams in clusters and cloud services. One of the first DSPEs to gain popularity is Storm [27]. It relies on topologies: processing workflows where each element is named task. Storm automatically handles the distribution of tasks to computing nodes. Recently, Twitter proposed Heron [13] as a successor of Storm. Heron overcomes some limitations of Storm: the limited performance monitoring, impossibility to deploy in clusters with heterogeneous nodes and complexity in debugging. Other DSPEs exist, each of those with different design goals but with the common idea of workflows, similarly to Apache Storm's topologies. For example, Apache Samza embeds a key-value store to manage state between processing nodes. Apache Flink [7] emphasizes the combination of batched-based and stream-based processing paradigms.

RDF Stream Processing. RDF Stream Processing (RSP) is a recent effort to push the stream processing paradigm in the semantic web. Solutions like CSPARQL [5] and CQELS [14] adopts sliding windows to create time-varying views over the streams, to be processed through SPARQL. Solutions like EPSPARQL [1] and INSTANS [25] propose to verify time-relation constraints over the stream elements, usually in closed time intervals. The existing RSP solutions have been developed in centralized systems, showing limitations in scalability and in managing data characterized by high velocity and volume. 


\section{Solution}

Given a Tbox, our solutions derive incremental consistency checking procedures to be executed in a DSPE. We first present the running example used in this section, followed by the basic building blocks used in our solutions.

Example 1. Fig. 2 shows the TBox $\mathcal{T}_{\text {ex }}$, used as running example and based on the LUBM benchmark. Each node in the figure is a class, while edges denote positive inclusion axioms (PIs), e.g., the axiom $S C$ (Student, Person) is represented by the nodes Student, Person and the edge between them. The right part of Fig. 2 contains the negative inclusion axioms (NIs) (e.g., Axiom (1) indicates that an instance of Student cannot be an instance of Publication). In total, there are ten classes, six PIs, and ten NIs that are explicitly stated in $\mathcal{T}_{\text {ex }}$.

A tuple is the basic unit of input, output, and intermediate streams. With reference to the RDF stream model of Section 2, each stream item is a tuple

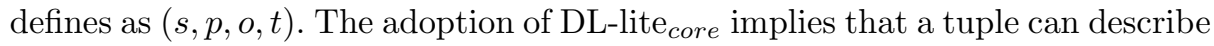
either a role or a class assertion. Class assertions $C(x)$ and $\exists R(x)$ in the snapshot $\mathcal{A}_{m}^{n}(t)$ are represented as $(x, i s A, C, t)$ and $\left(x, i s A, C_{R}, t\right)$, respectively.

A stream $S_{C}$ contains all tuples that state a class assertion of $C(a)$, where $a$ is a generic individual, e.g., $S_{\text {Person }}$ is a stream that has instances like (Bob, is A, Person, $\left.t_{1}\right)$. $S_{C_{i n c}}$ is a special stream that contains the instances found to be inconsistent, e.g., given Axiom (1) in $\mathcal{T}_{\text {ex }}$, if there are two instances $\left(B o b, i s A\right.$, Person, $\left.t_{1}\right)$ and (Bob, is A, Publication, $\left.t_{1}\right)$, the output tuple (Bob, isFoundToBe, Inconsistent, $\left.t_{1}\right)$ is appended to $S_{C_{i n c}}$. Role assertions $R(a, b)$ are managed by introducing two class assertions: $\exists R(a)$ and $\exists R^{-}(b)$, where $R^{-}$is the inverse property of $R$.

Given a TBox $\mathcal{T}$, the input to a topology is a set of streams $\mathcal{S}=\left\{S_{C_{1}}, \ldots, S_{C_{n}}\right\}$, where each stream corresponds to a class in $\mathcal{T}$. The output of the topology is

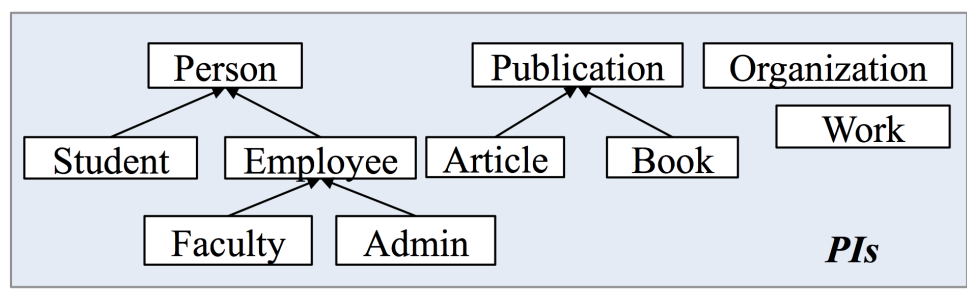

$$
\begin{aligned}
& \text { Person } \sqsubseteq \neg \text { Publication (1) Person } \sqsubseteq \neg \text { Organization (2) } \\
& \text { Person } \sqsubset \neg \text { Work (3) Publication } \sqsubseteq \neg \text { Organization (4) } \\
& \text { Publication } \sqsubset \neg \text { Work (5) Organization } \sqsubseteq \neg \text { Work (6) } \\
& \text { Student } \sqsubseteq \neg \text { Employee (7) Article } \sqsubseteq \neg \text { Book (8) } \\
& \text { Faculty } \sqsubseteq \neg A d m i n(9) \text { Faculty } \sqsubseteq \neg S t u d e n t(10) \text { NIs }
\end{aligned}
$$

Fig. 2: The PIs and NIs of the Tbox $\mathcal{T}_{\text {ex }}$ in the running example. 
$S_{C_{i n c}}$ that reports all inconsistent instances. For the sake of illustration, we assume that a topology has only one spout serving as a stream broker, i.e., it collects streams from different sources and emits them as $\mathcal{S}$.

A topology consists of a set of bolts $\mathcal{B}=\left\{B_{1}, \ldots, B_{n}\right\}$. A bolt $B_{i}$ encodes a set of operations $\left\{o_{1}, \ldots, o_{n}\right\}$. An operation $o_{i}$ encodes a part of the consistency check logics: it takes as input a set of streams $\mathcal{S}_{\text {input }}^{o_{i}}=\left\{S_{C_{1}}, \ldots, S_{C_{n}}\right\}$ and emits output streams $\mathcal{S}_{\text {output }}^{o_{i}}$. We define two kinds of operations. An inference operation $o \rightarrow$ encodes subclass to superclass inferences: it takes one stream $S_{C_{1}}$ as input and emits one output stream $S_{C_{2}}$, i.e., $o \rightarrow: S_{C_{1}}(x) \rightarrow S_{C_{2}}(x)$. A conjunction operation $o^{\cap}$ is similar to a join function that checks inconsistencies. The input is a set of streams and the output is one stream, i.e., $o^{\cap}: S_{C_{1}}(x) \wedge$ $\ldots \wedge S_{C_{n}}(x) \rightarrow S_{C_{\text {output }}}(x)$. Differently from inference operations, conjunction ones require the presence of several assertions together to trigger the underlying rule. Therefore, a conjunction operation requires caching instances of each input streams. We exploit windows for this purpose: they cache instances when they arrive and delete them when their associated time instant expires.

For both operations, the key should be the value of the instance, e.g., an instance of $S_{P e r s o n(B o b)}$ uses "Bob" as a key. This allows the engine to partition the streams and make sure that instances Person(Bob) and Publication(Bob) are processed in the same task instance. The operation output can not serve as input to another operation of the same bolt, i.e., the operations within one bolt are independent of each other, enabling the distribution of workload. Lastly, if an instance does not participate any operations, the bolt simply forwards it to the downstream bolts, which guarantee that all the inconsistencies are detected.

Example 2. Given the $\mathcal{T}_{\text {ex }}$ in Example 1, the inference operation $o_{i} \rightarrow$ derives a new instance of Person for each instance in the Student stream, since Student is a subclass of Person, i.e., $o_{i}:=S_{\text {Student }}(x) \rightarrow S_{\text {Person }}(x)$. Moreover, given the Axiom (1) in Fig. 2, the conjunction operation $o_{i}^{\cap}$ is defined as $o_{i}^{\bigcap}:=S_{P e r s o n}(x) \wedge$ $S_{\text {Publication }}(x) \rightarrow S_{C_{\text {inc }}}(x)$. In this case, each stream of $S_{\text {Person }}$ and $S_{\text {Publication }}$ is associated with a window. When an instance $S_{P e r s o n(B o b)}$ arrives, $o_{i}^{\cap}$ checks whether $S_{\text {Publication }(B o b)}$ is cached. If yes, $o_{i}^{\bigcap}$ outputs $S_{C_{\text {inc }}(B o b)}$, since it violates the NI Axiom (1); otherwise, Person $(B o b)$ is cached for future use.

\subsection{The NIs Topology Method (NTM)}

Fig. 3 depicts three alternative topologies that are compiled from $\mathcal{T}_{\text {ex }}$, produced by the two methods we propose. Fig. 3a shows our basic solution, the NIs Topology Method (NTM): it computes the set $N I_{\text {closure }}$, which contains all the disjointness axioms that can be deduced from the NIs and PIs. Then, it compiles each of them into one bolt as conjunction operations. Specifically, given an NI in the TBox, NTM computes $N I_{\text {closure }}$ as the list of subclasses for each class in the NI. The permutation of the subclass elements gives all possible combinations between subclasses, and hence the $N I_{\text {closure }}$. Each NI in $N I_{\text {closure }}$ is of the form $D J\left(C_{1}, \ldots, C_{n}\right)$, which can be implemented as a conjunction operation. NTM computes topology before runtime since it requires the TBox only. 


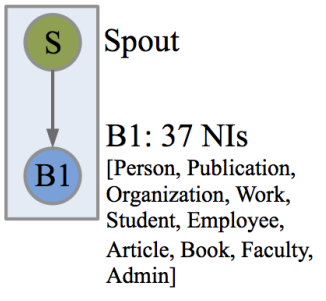

(a) NIs Topology

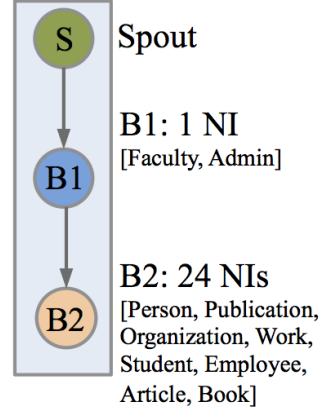

(b) Pipeline Topology 1

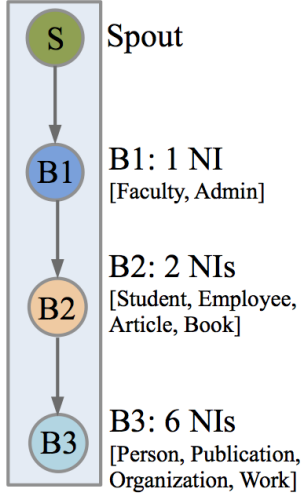

(c) Pipeline Topology 2

Fig. 3: The NIs (NTM) and the Pipeline (LTM) topologies. The workload in the last bolt of the three topologies reduces as the length of the pipeline increases.

Example 3. The ten NIs in $\mathcal{T}_{\text {ex }}$ of Fig. 1 lead to $37 \mathrm{NIs}$ in $N I_{\text {closure }}$ e.g., given DJ(Person, Work) and the subclasses of Person, NTM infers NIs that are not explicitly stated in $\mathcal{T}_{\text {ex }}$ : DJ(Person,Work), DJ(Employee, Work $)$, $D J($ Faculty, Work $)$, and DJ(Admin,Work). As shown in Fig. 3a, $B_{1}$ is the only bolt that performs all the conjunction operations. When deploying this topology, multiple task instances of the bolt partition the stream by the subject field in a tuple, e.g., given a tuple $(a, i s A, C, t), a$ is the partition key.

As shown in the experiment, the single bolt of NTM creates a computational bottleneck on the throughput. [6] explains that the size of $N I_{\text {closure }}$ is exponential to the size of TBox at worst. Consequently, the number of operations in the bolt may be exponential. We considered two possible remedies to the bottleneck problem, but neither of them solves it completely. A possible remedy is to increase the number of tasks for the bolt. It increases the parallelism, but it cannot reduce the complexity of the bolt. When the stream rate increases, the workload on each task increases as well, which poses the same problem. Another possible remedy is to have a different topology layout. Consider $\mathcal{T}_{\text {ex }}$ and a topology where the spout connects to two bolts $B_{1}$ and $B_{2}$ (not chained in a pipeline): it does not offer any advantages when processing the pairwise PIs between Person, Publication, Organization, and Work (Axioms (1-6)), since these four streams cannot be split into two bolts without duplication.

\subsection{The Pipeline Topology Method (LTM)}

We propose the Pipeline Topology Method (LTM) to improve NTM. LTM arranges the NIs in a hierarchical fashion to reduce their total number, and splits the hierarchy in different bolts to distribute the consistency-checking workload. 
The bolts in LTM are chained as a pipeline. Each bolt deals with a limited amount of classes and their related NIs. The example below shows our idea.

Example 4. In Fig. 3b, bolt $B_{1}$ is assigned to process only two classes Faculty and Admin, as well as the only NI between them. In addition, $B_{1}$ has two inference operations that convert these two streams into one Employee stream and output to bolt $B_{2}$. $B_{1}$ also forwards all other unused streams to $B_{2}$. In this way, $B_{2}$ does not need to consider Faculty and Admin anymore. Their related NIs with other classes are still ensured by the disjointness between Employee and other classes. Comparing to Fig. 3a, this topology has the cost of adding an extra bolt $B_{1}$, however, it greatly reduces the number of NIs in the last bolt from 37 to 24 . Assuming each class has the same incoming stream rate, the CPU bottleneck of the last bolt is significantly reduced. Furthermore, the total number of NIs in $B_{1}$ and $B_{2}$ together is also smaller than the number of NIs in Fig. 3a. The topology in Fig. 3c further extends this idea by using three bolts. Bolt $B_{2}$ handles DJ (Student, Employee) and DJ(Article, Book), while bolt $B_{3}$ handles the rest of the classes and the six NIs among them. The topology in Fig. 3c has the overhead of forwarding streams through $B_{1}$ and $B_{2}$.

By comparing the three topologies in Fig. 3, we observe that the excessive amount of NIs in $B_{1}$ of Fig. 3a can lead to a computational bottleneck. By arranging the NIs in a hierarchal way, the total number of NIs are significantly reduced. It is possible to use one bolt to hold the entire reduced NI hierarchy. However, it still has the same problem that the single bolt can become a CPU hot-spot. Therefore, by using the operations we defined in Section 2, we split the hierarchy into different bolts, such as in Fig. 3b and 3c. In this way, we achieve that the CPU cost of checking NIs is distributed along the pipeline and alleviate the potential computational bottleneck. Essentially, our LTM method looks for the best way of balancing the workload into a pipeline of bolts.

Algorithm 1 gives the details of LTM. The algorithm takes the TBox $\mathcal{T}$ of the DL ontology stream as an input. The output is a chain (pipeline) of bolts. Each bolt is filled with the operations it needs to perform. The algorithm develops two parts: first, Steps 1-3 find the essential NIs and arrange them into hierarchical groups. Steps 4-5 assign these groups to bolts and generate the corresponding operations. Each step of Algorithm 1 is explained in below.

Step 1. As with NTM, Line 1 computes the NI closure set, denoted as $N I_{\text {closure }}$.

Step 2. Based on $N I_{\text {closure }}$, Line 2 computes $N I_{\text {root }}$, which is a set of all essential NIs. The intuition of "essentialness" is that: given two NIs, if each class in one NI is either a sub or an equivalent class of the other, we can first convert the subclass to its superclass, then only process the NI with the superclasses. For example, given Axiom (7) and (10) of Fig. 2, Faculty in Axiom (10) is a subclass of Employee in Axiom (7). Therefore, we can first make an inference operation (i.e., $o_{i}$ : Faculty $\rightarrow$ Employee), and then check Axiom (7) only. By iterating through each pair of NIs in $N I_{\text {closure }}$, we can find and keep the essential NIs in $N I_{\text {root }}$. Among the ten NIs in Fig. 2, Axiom (10) is removed from $N I_{\text {root }}$. 


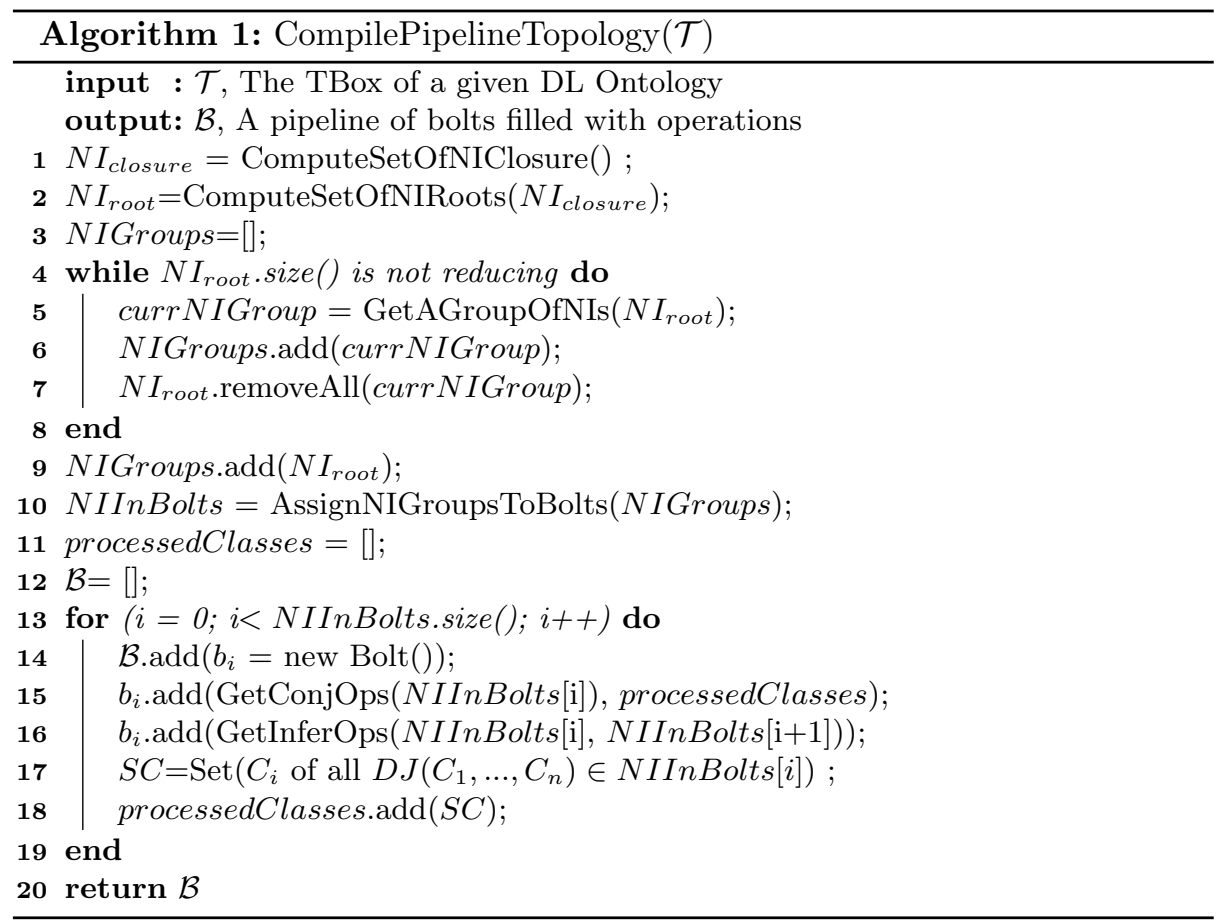

Step 3. Lines 4-8 of Algorithm 1 group NIs into a sequential processing order. Intuitively, an NI with no subclasses can be checked first. For example, classes of NI Axiom (9) have no subclasses. It should be checked at the beginning of a pipeline topology (e.g., Place it in bolt $B_{1}$ of Fig. 3c. If placed in $B_{3}$, its input streams need to be forwarded via $B_{1}$ and $B_{2}$ ). These NIs can be found by counting the "in-degree". For example, in Fig. 4a, if there is a sub-to-super relationship between classes of two NIs, we draw an edge between them. The NIs with no incoming edges have zero in-degree. However, not all NIs with zero in-degree should be processed first. For example, DJ(Organization, Work) cannot be processed in bolt $B_{1}$ of Fig. 3c, since both Organization and Work are used again later in the pipeline by other NIs. If placed in $B_{1}$, every tuple of $S_{\text {Organization }}$ still needs to be forwarded to $B_{3}$ for the Axiom (2) DJ(Organization, Person). Therefore, DJ(Organization, Work) is placed together with Axiom (2) at the last group in Fig. 4a. In total, there are three NI groups in Fig. 4a that should be checked in a sequential order.

The algorithm of forming NI groups is adapted from the topological sorting algorithm. It first finds all the NIs in $N I_{\text {roots }}$ that fulfill both conditions: 1) has zero in-degree; and 2) its classes are used by the NIs outside the group. These NIs are removed from $N I_{\text {roots }}$ and form an NI group. Then, the algorithms continue to find another group from $N I_{\text {roots }}$ until no group can be found. 


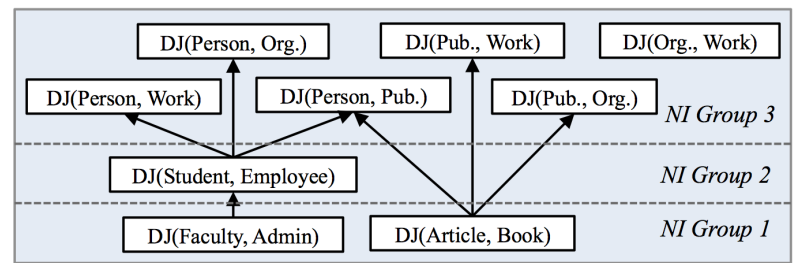

(a) NI groups

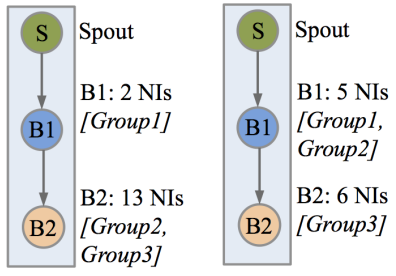

(b) Combining NI groups

Fig. 4: Computing NI groups and assigning them to bolts

Step 4. After Step 3, each NI group can individually form a bolt in the pipeline. This, however, may create too many bolts, and each new bolt incurs extra overhead. To avoid this problem, several adjacent NI groups can be combined into one bolt (Line 10). Fig. 4 gives two ways of combining NI groups to bolts. Fig. $4 \mathrm{a}$ contains three NI groups separated by the dashed line. In Fig. 4b, the left topology assigns Group 1 to bolt $B_{1}$, and Group 2 and 3 to bolt $B_{2}$. The right topology has Group 1 and 2 assigned to $B_{1}$, and Group 3 to $B_{2}$. The way of combining NI groups impacts system performance, as we discuss at the end of this section.

Step 5. Finally, Line 12-20 compiles the operations $\left(o^{\rightarrow}\right.$ and $\left.o^{\cap}\right)$ in each bolt.

To compile conjunction operations $o^{\cap}$, we compute the closure of NIs in a group to ensure correctness. For example, in the right topology of Fig. 4b, since Group 1 and 2 are combined in bolt $B_{1}$, the algorithm first computes their NI closure and then checks each of them (e.g., the closure contains NIs from the two groups together with DJ(Student, Faculty) and DJ(Student, Admin)). The process of computing an NI closure has been discussed in NTM. The closure also excludes the classes that have processed in the preceding bolts.

For compiling inference operations $o \rightarrow$, we need to find the subclass axioms between the classes of two consecutive bolts $\left(B_{i}\right.$ and $\left.B_{i+1}\right)$. For example, in the left topology in Fig. $4 \mathrm{~b}$, bolt $B_{1}$ should include the subclass axioms between $B_{1}$ and $B_{2}$ (e.g., $S C$ (Faculty, Employee) and $S C($ Admin, Employee)). We consider only the direct subclass axioms. Axioms like $S C$ (Faculty, Person) are not included, since $B_{2}$ handles the NIs between Employee and all other classes. Furthermore, bolt $B_{1}$ also needs to include all the subclasses of the classes in $B_{2}$ that do not participate in the NI roots. For example, if class Employee (handled in $B_{2}$ ) has a subclass FemaleEmployee, which is not disjoint with either Faculty or Admin, it still needs to be inferred at bolt $B_{1}$ to become Employee so that the disjointness between FemaleEmployee and other classes are checked in $B_{2}$. Each of these subclass axioms can be compiled to an inference operation. Lastly, Algorithm 1 returns a list of bolts that forms a pipeline. Each bolt is filled with the necessary operations to check the consistency.

As mentioned in Step 4, the two ways of combining NI groups have different performance. Based on the actual number of NIs we derived in Step 5 for Fig. 4b, 
it follows that the right topology distributed the workload more evenly than the left one. The difference of NIs numbers between $B_{1}$ and $B_{2}$ is much less on the right topology. In the left topology, $B_{2}$ will become a bottleneck for the throughput assuming all input streams have the same stream rate. In practice, combining NI groups to bolts is a parameter to be specified by the users. To find the best way of combing NI groups, users can compile different topologies beforehand and choose the one with less skewed workload based on the number of operations. In future, we will extend our work to propose a cost model, where the cost of each operation, the stream rate, the computation, and communication overhead will be considered together to automatically find the best topology.

\subsection{Limitations and Discussion}

This study considers DL-lite core $_{\text {. One of its closest extensions is DL-lite }}$ horn [3], which introduces the conjunction of concepts in the left operator, i.e. $C_{1} \sqcap \ldots \sqcap$ $C_{n} \sqsubseteq C$. The conjunction operation defined above supports this type of axioms. Our above algorithm needs to be extended to cope with it.

LTM has a relatively high memory cost comparing with NTM. The reason is that an instance of a stream can be potentially cached multiple times at different bolts of a LTM topology. For example, in Fig. 3b, stream $S_{\text {Faculty }}$ needs to be cached in $B_{1}$, since $B_{1}$ checks its NI with $S_{\text {Admin }}$. After stream Faculty is inferred as Employee and sent to $B_{2}$, its instances need to be cached again in $B_{2}$. Our experiments also show this shortcoming. LTM should consider the memory limitation when assigning NI groups to bolts. This work assumes enough memory to emphasize the CPU cost.

\section{Experiments}

This section first introduces the experiment setup and then report the results.

Setup. As in [28], we use two ontologies for experiment, LUBM [11] and NPD [26].

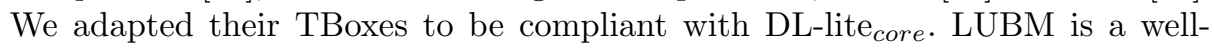
established benchmark, while NPD is an ontology from the real world. NPD is larger than LUBM: there are 43 streams in the input stream set for LUBM and 329 of them for NPD. In the adapted TBox, LUBM has 56 PIs and 70 NIs; NPD has 332 PIs and 51 NIs. Although NPD has less NIs its closure is bigger than the LUBM's one. We used HermiT [10] to calculate the closure set of PIs.

Both LTM and NTM have one spout, as discussed in Section 4 . The generator emits each stream at the same speed, fine tuned to target the system processing capability. We compare the throughput of the topologies by measuring the number of tuples they process in a time interval. We use Heron 0.14.3 as the DSPE; experiments run with 4-6 machines, each of them having 128 GB RAM and two E5-2680 v2 at $2.80 \mathrm{GHz}$ processors, with 10 cores per processor. 


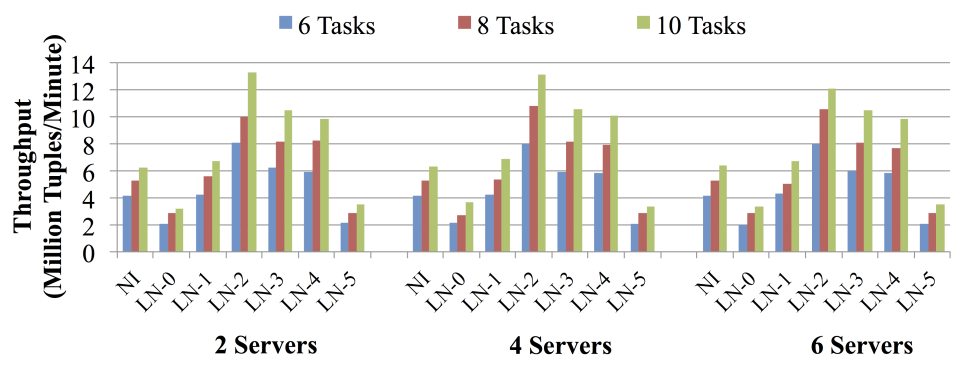

(a) Results of LUBM benchmark

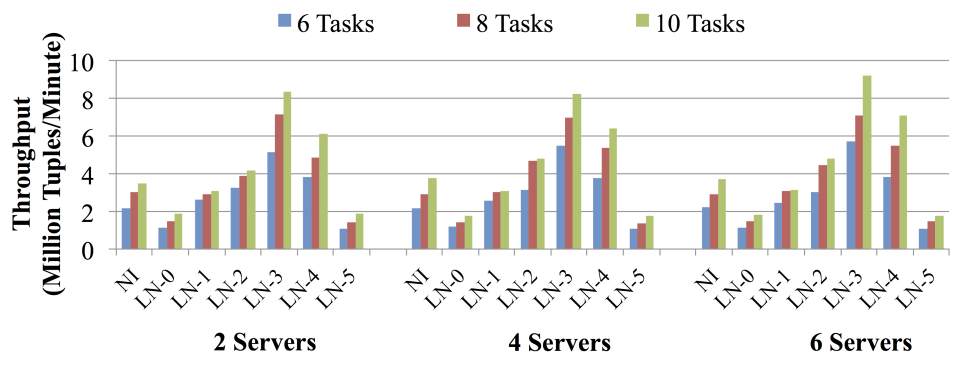

(b) Results of NPD benchmark

Fig. 5: Results of a NTM topology and LTM topologies with two bolts. The y axis gives the throughput (number of tuples per minutes); the $\mathrm{x}$ axis denotes the topologies: NTM stands for the NTM topology, LTM- $n$ denotes a LTM topology.

Overall results. Fig. 5 plots the results for LUBM and NPD. We compare the topology throughput by using two, four, and six computing servers and two bolts for LTM, i.e., LTM topologies have two bolts, $B_{1}$ and $B_{2}$. A topology LTM- $n$ has the first $n$ NI groups assigned to $B_{1}$. Recall that given the three NI groups in Fig. 4a, the LTM-2 topology has the first two NI groups assigned to $B_{1}$ and the others to $B_{2}$, which results in the right topology in Fig. 4b. Note that LTM-0 places no NI groups in bolt $B_{1}$, and has $B_{2}$ to check all the NIs. The compiled TBoxes of LUBM and NPD happen to have the same number of five NI groups.

Throughputs are compared under the same amount of tasks for both topologies, e.g. if they are set to have six tasks, in NTM (Fig. 3a) $B_{1}$ will have six tasks; in LTM, $B_{1}$ and $B_{2}$ will have three tasks each (Fig. 3b).

Let's consider the results of LUBM in Fig. 5. First, when using two servers, the throughput of NTM is about twice as much as LTM-0 under different task numbers. Since $B_{1}$ in LTM-0 contains no NIs, its three tasks only forward streams to $B_{2}$. The three tasks of $B_{2}$ in LTM-0 bear the same workload of the six tasks of bolt $B_{1}$ in NTM. This explains why the throughput of LTM-0 is roughly half of NTM one. Second, we can observe that LTM-1 greatly improves the throughputs of LTM-0 under different task numbers. This is because LTM-1 distributes the NI-checking workload between $B_{1}$ and $B_{2}$. Furthermore, the total number of NIs is also reduced, when arranging them in a pipeline. However, the 


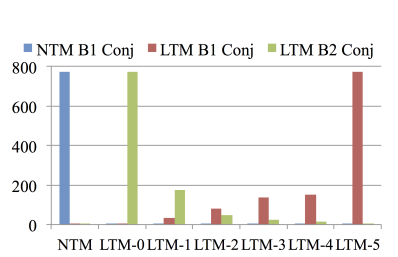

(a) Operations breakdown

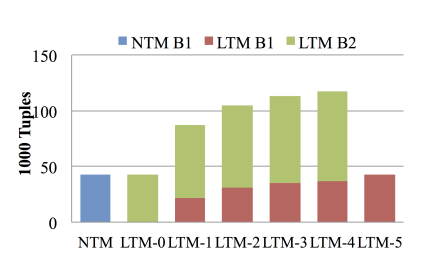

(b) Memory cost

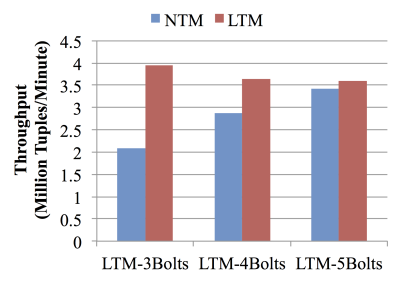

(c) LTM with $\mathrm{n}$ bolts

Fig. 6: Conjunction operations breakdown, memory cost, and LTM with n bolts.

throughputs of NTM and LTM-1 are roughly the same. This suggests that the way LTM-1 distributes the workload cannot outperform NTM. Given the same amount computing resources, $B_{2}$ in LTM-1 is still the bottleneck. Third, LTM2 shows the best performance: it outperforms NTM by up to $139 \%$ with six tasks. This suggests that LTM-2 topology distributes NIs more evenly (there are two NI groups assigned to $B_{1}$ of LTM-2) than LTM-0 and LTM-1, and LTM can perform better than NTM. Fourth, moving from LTM-2 to LTM-5 the throughput decreases, since more NI groups are placed onto $B_{1}$ than on $B_{2}$ and lead to an unbalanced situation. LTM-5 has all NI groups assigned to $B_{1}$, therefore, its performance is similar to that of LTM-0. The change of throughput from LTM-0 to LTM-5 shows that evenly distributing the NI-checking workload can avoid the bottleneck bolt slowing down the overall performance.

Looking at NPD in Fig. 5, the trend of throughput is similar to LUBM: it grows from LTM-0 to LTM-3 and then decreases. NPD always has the best performance at LTM-3 topology: it follows that LTM-3 has the most evenly distributed workload. The throughput improvement of LTM over NTM in the NPD case is up to $330 \%$, when LTM-3 topology has ten tasks running on six servers. The improvement ratio is higher than that of the LUBM case: the reason is that NPD has a much larger TBox. When arranging its NIs into a hierarchy, LTM saves more conjunction operations by replacing them with inference operations.

Operations Breakdown and Memory Cost. Fig. 6a and 6b plot two metrics to further investigate the reasons of the above results. Since the results are similar on LUBM and NPD, we focus on the former for brevity. Fig. 6 a gives the breakdown of the number of conjunction operations in each bolt. NTM, LTM-0, and LTM-5 have the same amount of 773 conjunction operations (NIs) executed in one bolt, since the closure of NIs is computed by using all the classes. The plot shows that the conjunction number of $B_{1}$ (green bar) decreases as the number of $B_{2}$ increases (red bar). LTM-2 has the smallest difference between $B_{1}$ and $B_{2}$, suggesting the reasons for its best throughput. The number of inference operations is relatively small (around 20), so it is not plotted in the figure.

Fig. 6b reports the memory cost (as the number of tuples cached). NTM, LTM-0 and LTM-5 have the same memory footprint, since they have similar 
NTM workloads. LTM-(1-4) incur higher memory costs than NTM, LTM-0 and LTM-5. As discussed in Section 4.3, it reflects one shortcoming of LTM that some instances need to be cached multiple times in the pipeline, e.g., in Fig. 3b, an instance of Faculty is cached at $B_{1}$, and after $B_{1}$ infers Employee, the instance needs to cached again in $B_{2}$. Furthermore, the memory footprint grows from LTM-1 to LTM-5. As $B_{1}$ handles more classes and inference operations, more instances need to be cached in $B_{2}$. Hence, the total memory cost grows.

Results of LTM with multiple bolts. Fig. 6c compares NTM and LTM topologies with different number of bolts (using three servers). We increase the bolts number for LTM from three to five (each bolt has one task). The task number of $B_{1}$ in NTM is set to be three, four, and five. We fine-tuned the NI groups for LTM to give the best performance. Fig. 6c shows that the throughput of LTM decreases when using more bolts. This is because the communication cost increases. Moreover, the throughput of NTM increases linearly with the number of tasks. This suggests that the benefits of reducing NIs might be offset by the communication cost when the length of a pipeline topology grows.

\section{Conclusions}

While consistency checking is usually studied in the context of static knowledge bases, in this paper, we focus on the problem of incremental consistency checking over streams and propose scalable solutions that can be deployed on a DSPE. More precisely, our two methods can compile a DL-lite core $_{\text {TBox into a processing }}$ workflow of a DSPE. The baseline method NTM adapts techniques such as NIs query rewriting to generate continuous queries to assess the consistency. However, NTM involves an excessive number of operations that slow down its

performance. To overcome this issue, we propose LTM, where the workload of consistency checking is distributed across a pipeline. This leads to a reduction of the CPU overhead and an improvement of the throughput. Our experiment results show that LTM outperforms NTM by up to $139 \%$ and $330 \%$, based on the LUBM benchmark and the NPD ontology, respectively.

\section{References}

1. Anicic, D., Fodor, P., Rudolph, S., Stojanovic, N.: EP-SPARQL: a unified language for event processing and stream reasoning. In: WWW. pp. 635-644. ACM (2011)

2. Arenas, M., Bertossi, L.E., Chomicki, J.: Consistent Query Answers in Inconsistent Databases. In: PODS. pp. 68-79. ACM Press (1999)

3. Artale, A., Calvanese, D., Kontchakov, R., Zakharyaschev, M.: The dl-lite family and relations. CoRR abs/1401.3487 (2014)

4. Baclawski, K., Kokar, M.M., Waldinger, R.J., Kogut, P.A.: Consistency checking of semantic web ontologies. In: ISWC '02. pp. 454-459. ISWC '02 (2002)

5. Barbieri, D.F., Braga, D., Ceri, S., Della Valle, E., Grossniklaus, M.: Querying RDF streams with C-SPARQL. SIGMOD Record 39(1), 20-26 (2010) 
6. Botoeva, E., Artale, A., Calvanese, D.: Query Rewriting in DL-Lite ${ }_{\text {horn }}^{(H N)}$. In: Description Logics. CEUR Workshop Proceedings (2010)

7. Carbone, P., Katsifodimos, A., Ewen, S., Markl, V., Haridi, S., Tzoumas, K.: Apache Flink ${ }^{\mathrm{TM}}$ : Stream and Batch Processing in a Single Engine. IEEE Data Eng. Bull. 38(4), 28-38 (2015)

8. Cugola, G., Margara, A.: Processing flows of information: From data stream to complex event processing. ACM Comput. Surv. 44(3), 15 (2012)

9. Flouris, G., Huang, Z., Pan, J.Z., Plexousakis, D., Wache, H.: Inconsistencies, Negations and Changes in Ontologies. In: AAAI2006. pp. 1295-1300 (2006)

10. Glimm, B., Horrocks, I., Motik, B., Stoilos, G., Wang, Z.: HermiT: An OWL 2 Reasoner. J. Autom. Reasoning 53(3), 245-269 (2014)

11. Guo, Y., Pan, Z., Heflin, J.: LUBM: A benchmark for OWL knowledge base systems. J. Web Sem. 3(2-3), 158-182 (2005)

12. Kontchakov, R., Lutz, C., Toman, D., Wolter, F., Zakharyaschev, M.: The combined approach to query answering in dl-lite. pp. 247-257. KR'10 (2010)

13. Kulkarni, S., Bhagat, N., Fu, M., Kedigehalli, V., Kellogg, C., Mittal, S., Patel, J.M., Ramasamy, K., Taneja, S.: Twitter Heron: Stream Processing at Scale. In: SIGMOD. pp. 239-250 (2015)

14. Le Phuoc, D., Dao-Tran, M., Parreira, J.X., Hauswirth, M.: A native and adaptive approach for unified processing of linked streams and linked data. In: ISWC'11. pp. 370-388 (2011)

15. Lecue, F., Pan., J.Z.: Consistent Knowledge Discovery from Evolving Ontologies. In: Proc. of AAAI'15. (2015)

16. Lembo, D., Ruzzi, M.: Consistent Query Answering over Description Logic Ontologies. In: Description Logics (2007)

17. Pan, J., Calvanese, D., Eiter, T., Horrocks, I., Kifer, M., Lin, F., Zhao, Y.: Reasoning Web: Logical Foundation of Knowledge Graph Construction and Querying Answering. Springer (2017)

18. Pan, J., Vetere, G., Gomez-Perez, J., Wu, H.: Exploiting Linked Data and Knowledge Graphs for Large Organisations. Springer (2016)

19. Paulheim, H., Gangemi, A.: Serving dbpedia with dolce - more than just adding a cherry on top. In: ISWC. pp. 180-196 (2015)

20. Paulheim, H., Stuckenschmidt, H.: Fast approximate a-box consistency checking using machine learning. In: ISWC. pp. 135-150 (2016)

21. Pérez-Urbina, H., Motik, B., Horrocks, I.: A comparison of query rewriting techniques for dl-lite. In: Proceedings of DL'09 (2009)

22. Ren, Y., Pan, J.Z.: Optimising Ontology Stream Reasoning with Truth Maintenance System. In: CIKM2011 (2011)

23. Ren, Y., Pan, J.Z., Guclu, I., Kollingbaum, M.: A Combined approach to Incremental Reasoning for EL Ontologies. In: RR2016. pp. 167-183 (2016)

24. Ren, Y., Pan, J.Z., Guclu, I., Kollingbaum, M.J.: A combined approach to incremental reasoning for EL ontologies. In: RR'16. pp. 167-183 (2016)

25. Rinne, M., Solanki, M., Nuutila, E.: Rfid-based logistics monitoring with semanticsdriven event processing. In: DEBS. pp. 238-245 (2016)

26. Skjæveland, M.G., Lian, E.H., Horrocks, I.: Publishing the norwegian petroleum directorate's factpages as semantic web data. In: ISWC. pp. 162-177 (2013)

27. Toshniwal, A., Taneja, S., Shukla, A., Ramasamy, K., Patel, J.M., Kulkarni, S., Jackson, J., Gade, K., Fu, M., Donham, J., Bhagat, N., Mittal, S., Ryaboy, D.V.: Storm@twitter. In: SIGMOD. pp. 147-156 (2014)

28. Wu, J., Lécué, F.: Towards Consistency Checking over Evolving Ontologies. In: CIKM. pp. 909-918. ACM (2014) 